\title{
Assessment of intrathoracic lymph nodes by FDG PET/CT in patients with asbestos-related lung cancer
}

\author{
Norichika Iga $^{1}$, Hiroshi Sonobe ${ }^{2}$, Daisuke Mizuno ${ }^{1}$, Hideyuki Nishi $^{1}$ \\ ${ }^{1}$ Department of Surgery, Okayama Rosai Hospital, Chikkomidorimachi, Minamiku, Okayama, Japan; ${ }^{2}$ Department of Pathology, Okayama Rosai \\ Hospital, Chikkomidorimachi, Minamiku, Okayama, Japan \\ Contributions: (I) Conception and design: N Iga, H Sonobe; (II) Administrative support: D Mizuno, H Nishi; (III) Provision of study materials or \\ patients: N Iga, H Nishi; (IV) Collection and assembly of data: N Iga; (V) Data analysis and interpretation: N Iga, H Sonobe; (VI) Manuscript \\ writing: All authors; (VII) Final approval of manuscript: All authors. \\ Correspondence to: Norichika Iga. Department of Surgery, Okayama Rosai Hospital, 1-10-25 Chikkomidorimachi, Minamiku, Okayama 702-8055, \\ Japan. Email: igatoku0613@yahoo.co.jp.
}

Background: This study explored the assessment of intrathoracic lymph node metastasis by 18F-fluoro-2deoxy-D-glucose (FDG) positron emission tomography computed tomography (PET/CT) in patients with asbestos-related lung cancer (ARLC).

Methods: We retrospectively reviewed the data on 35 patients with ARLC who underwent preoperative FDG-PET/CT and surgical resection between January 2012 and December 2018. We collected medical information from medical records and imaging systems and examined the FDG uptake in each lymph nodal region resected by surgery and the presence or absence of pathological lymph node metastasis.

Results: Pathological lymph node metastases were detected in 14 (8.70\%) of 161 nodal stations. The sensitivity, specificity, positive predictive value, negative predictive value, and accuracy of FDG-PET/CT were 71.4\% (10/14), 87.8\% (129/147), 35.7\% (10/28), 97.0\% (129/133), and 86.3\% (139/161), respectively. Six of the eight false-positive patients had bilateral accumulations, whereas all six true-positive patients had unilateral accumulation $(\mathrm{P}=0.006)$. On histopathological examination, the false-positive nodes showed disruption of lymphoid follicles in the cortex, infiltration of histiocyte-like cells in the medulla, fibrous micronodules, and severe anthracosis.

Conclusions: PET/CT scans of patients with ARLC showed comparable sensitivity and specificity to those of PET/CT scans of patients with conventional lung cancer reported in the literature. Many falsepositive cases also showed bilateral symmetric accumulation. This method can be used to evaluate lymph node involvement in lung cancer.

Keywords: Positron emission tomography computed tomography (PET/CT); asbestos related lung cancer; false positive

Submitted Jun 12, 2021. Accepted for publication Aug 26, 2021.

doi: $10.21037 /$ jtd-21-974

View this article at: https://dx.doi.org/10.21037/jtd-21-974

\section{Introduction}

The diagnosis of hilar and mediastinal lymph node metastases in primary lung cancer is important for determining the indications for surgery, surgical techniques, and treatment options, including chemotherapy and radiation therapy. ${ }^{18}$ F-fluoro-2-deoxy-D-glucose
(FDG) positron emission tomography (PET) computed tomography (CT) is generally performed for nodal and systemic evaluation in conjunction with enhanced $\mathrm{CT}$ as a non-invasive modality. However, increased false-positive (FP) findings combined with pneumoconiosis, such as silicosis, may occur.

Asbestos is an occupational dust that causes lung cancer 
and malignant pleural mesothelioma. There are limited clinicopathological studies on asbestos-related lung cancer (ARLC), and mediastinal lymph node evaluation by FDGPET/CT in patients with ARLC has not been reported. Thus, this study explored the accuracy of FDG-PET/ CT for intrathoracic lymph node assessment in patients with ARLC compared to that of surgical and histological findings.

We present the following article in accordance with the STROBE reporting checklist (available at https://dx.doi. org/10.21037/jtd-21-974).

\section{Methods}

\section{Patients and data collection}

In Japan, the definition of ARLC is primary lung cancer with (I) asbestosis on chest radiography; (II) pleural plaques with $>10$ years of occupational asbestos exposure; (III) asbestos particles or fibers on the lung tissues with $>10$ years of occupational asbestos exposure; and (IV) >5,000 asbestos bodies per gram of dry lung tissue with occupational asbestos exposure (1).

This study included 35 patients with ARLC who underwent preoperative FDG-PET/CT and surgical resection between January 2012 and December 2018. We collected information from medical records and imaging systems and examined FDG accumulation in lymph node regions resected by surgery and the presence or absence of pathological lymph node metastasis. This retrospective observational study was conducted in accordance with the Declaration of Helsinki (as revised in 2013), and approved by our institutional review board (approval no. 167; approval date: February 29, 2019). Informed consent was waived because of the anonymous nature of the data.

\section{FDG-PET/CT image analysis}

FDG-PET/CT was performed in two medical institutions by their radiologists and in our institution by two thoracic surgeons. Intrathoracic lymph node stations were assessed according to the International Association for the Study of Lung Cancer lymph node map (2). Intrathoracic lymph nodes with focally increased FDG uptake, excluding physiologic uptake, compared to that in the surrounding normal tissue were considered positive.

\section{Surgery and histopathology}

All patients underwent standard hilar and mediastinal lymph node resection and pulmonary resection, including lobectomy and segmentectomy. The surgeons labeled the dissected lymph nodes by numbering them according to the lymph node map. Pathological review (primary tumor characteristics and lymph node status) was performed using standard techniques, and immunohistochemistry was used when appropriate.

\section{Statistical analysis}

FDG-PET/CT findings were compared to histological findings in the resected lymph node stations. Each lymph node station was classified as true positive (TP), true negative, FP, or false negative. All calculations and statistical tests were performed using StatMate version 5 (ATMS Publishing, Japan). The continuous variables were expressed as median [interquartile range (IQR) 25 th-75th percentile]. The categorical data were expressed as counts and proportions. The continuous variables were checked using the Mann-Whitney test, while the categorical variables were evaluated using the Fisher's exact test. $\mathrm{P}$ values $<0.05$ were considered statistically significant.

\section{Data analysis}

The FDG-PET/CT findings were compared to the histological findings in the resected lymph node stations to determine their diagnostic sensitivity and specificity. The sensitivity, specificity, and accuracy of integrated PET/CT in the assessment of intrathoracic lymph node involvement were determined using histological findings as the reference standard.

\section{Results}

\section{Pathology and nodal staging}

The characteristics of the enrolled patients are shown in Table 1 . The study group included 35 men with a mean age of 73 years [interquartile range (IQR): 70-78]. All patients had been exposed to asbestos but not to coal or silica; chest CT showed no evidence of asbestosis and no history of inflammatory lung diseases such as interstitial pneumonia 
Table 1 Patient characteristics

\begin{tabular}{|c|c|}
\hline Characteristics & $\mathrm{n}=35$ \\
\hline Age (years), median (IQR) & $73[70-78]$ \\
\hline \multicolumn{2}{|l|}{$\mathrm{BI}$} \\
\hline Median (IQR) & $800[505-980]$ \\
\hline Never & 3 \\
\hline \multicolumn{2}{|l|}{ Smoking habit } \\
\hline Ever & 32 \\
\hline $\mathrm{BI}>600$ & 22 \\
\hline \multicolumn{2}{|c|}{ Asbestos body/g dry lung tissue } \\
\hline Median (IQR) & $13,061(6,039-61,097)$ \\
\hline \multicolumn{2}{|l|}{ Tumor location } \\
\hline Right upper lobe & 10 \\
\hline Right middle lobe & 0 \\
\hline Right lower lobe & 10 \\
\hline Left upper lobe & 9 \\
\hline Left lower lobe & 6 \\
\hline \multicolumn{2}{|l|}{ Histology } \\
\hline Ad & 25 \\
\hline $\mathrm{Sq}$ & 8 \\
\hline $\mathrm{Ad}+\mathrm{Sq}$ & 1 \\
\hline carcinoid & 1 \\
\hline \multicolumn{2}{|l|}{ p-Nodal stages } \\
\hline NO & 26 \\
\hline N1 & 5 \\
\hline N2 & 4 \\
\hline \multicolumn{2}{|l|}{$\mathrm{p}$-Stage } \\
\hline 0 & 1 \\
\hline IA1/IA2/IA3/IB & $3 / 5 / 3 / 9$ \\
\hline IIA/IIB & $2 / 8$ \\
\hline IIIA/IIIB & $3 / 1$ \\
\hline
\end{tabular}

$I Q R$, interquartile range; $\mathrm{BI}$, Brinkman index; $\mathrm{Ad}+\mathrm{Sq}$, adenosquamous cell carcinoma; Ad, adenocarcinoma; Sq, squamous cell carcinoma.

or tuberculosis. The median smoking index was 800 , and 22 patients $(62.9 \%)$ were heavy smokers with smoking indices of $>600$. Asbestos bodies per gram of dry lung tissue representing asbestos exposure ranged from 6,039 to 61,097
Table 2 Occupational history

\begin{tabular}{lc}
\hline Items & No. \\
\hline Dockyard & 13 \\
Construction & 5 \\
Pipework & 5 \\
Brickyard & 3 \\
Electrical work & 2 \\
Steelwork & 2 \\
Machinery maintenance & 2 \\
Automobile maintenance & 1 \\
Metal casting & 1 \\
Textile processing & 1 \\
Industrial waste disposal & 1 \\
\hline
\end{tabular}

(interquartile range), with a median of 13,061.

Regarding occupational histories, 13 worked in dockyards, five in the construction industry, five in pipework, followed by brickyards and others, which are shown in Table 2. In this study, 25 (71.4\%) patients had adenocarcinoma and $8(22.9 \%)$ patients had squamous cell carcinoma. A total of 161 lymph node stations were dissected, including 18 superior mediastinal, 13 aortic, 32 inferior mediastinal, and 98 N1 lymph nodes. Pathological lymph node metastases were detected in 14 (8.70\%) of 161 nodal stations (Tables 3,4).

PET/CT correctly detected 10 metastatic lymph node stations (71.4\%; nine N1 and one N2). False-negative results were observed in four nodal stations (one N1 and three N2) and false-positive results were observed in 18 nodal stations (four N1 and $14 \mathrm{~N} 2$ ). The relationships between PET/CT lymph node evaluation and pathology at each station are shown in Tables 3,4. The overall sensitivity, specificity, positive predictive value, negative predictive value, and accuracy of FDG-PET/CT were $71.4 \%(10 / 14)$, $87.8 \%$ (129/147), 35.7\% (10/28), 97.0\% (129/133), and $86.3 \%(139 / 161)$, respectively.

\section{Characteristics of the false-positive lymph nodes}

The occupational history of the eight patients with falsepositive lymph nodes included four dockyards, one brickwork, one electrical work, one casting, and one pipework. Eighteen false-positive nodal stations were 
Table 3 Diagnosis by PET/CT and pathology of each lymph node station

\begin{tabular}{|c|c|c|c|c|c|c|c|c|c|c|}
\hline \multirow{2}{*}{$\begin{array}{l}\text { PET/CT diagnosis } \\
\text { Station No. }\end{array}$} & \multicolumn{4}{|c|}{ Superior } & \multicolumn{5}{|c|}{ Inferior } & \multirow{2}{*}{ Tota } \\
\hline & 2 & 4 & 5 & 6 & 7 & 9 & 10 & 11 & 12 & \\
\hline TP & 0 & 0 & 0 & 0 & 1 & 0 & 0 & 7 & 2 & 10 \\
\hline FN & 0 & 0 & 0 & 0 & 3 & 0 & 0 & 1 & 0 & 4 \\
\hline $\mathrm{TN}$ & 5 & 11 & 11 & 1 & 15 & 3 & 20 & 22 & 32 & 129 \\
\hline Total & 5 & 13 & 12 & 1 & 19 & 3 & 22 & 37 & 39 & 161 \\
\hline
\end{tabular}

TP, true positive; FN, false negative; FP, false positive; TN, true negative.

Table 4 PET/CT analysis with pathologic diagnosis

\begin{tabular}{|c|c|c|c|}
\hline \multirow{2}{*}{ Variable } & \multicolumn{3}{|c|}{ Number of lymph node stations } \\
\hline & LN meta (+) & LN meta $(-)$ & Total \\
\hline \multicolumn{4}{|l|}{$\mathrm{PET} / \mathrm{CT}^{*}$} \\
\hline Positive & 10 & 18 & 28 \\
\hline Negative & 4 & 129 & 133 \\
\hline Total & 14 & 147 & 161 \\
\hline \multicolumn{4}{|c|}{$\begin{array}{l}\text { *, sensitivity: } 71.4 \% \text {, specificity: } 87.8 \% \text {, PPV: } 35.7 \% \text {, NPV: } \\
97.0 \% \text {, accuracy: } 86.3 \% \text {. PPV, positive predictive value; NPV, } \\
\text { negative predictive value; LN meta (+), pathological lymph node } \\
\text { metastasis. }\end{array}$} \\
\hline
\end{tabular}

identified from eight patients, including 14 N1, 2 superior mediastinal, 1 aortic, and 1 inferior mediastinal lymph nodes. The short diameter of the lymph nodes on chest CT ranged from 5.3 to $6.7 \mathrm{~mm}$ (interquartile range), with a median of $6.3 \mathrm{~mm}$. Lymphadenopathy exceeding $10 \mathrm{~mm}$ in short diameter was observed in only 1 of the 18 stations (Tables 5,6). Histopathological examination of the FP nodes showed disruption of the lymphoid follicles in the cortex, infiltration of histiocyte-like cells in the medulla, fibrous micronodules, and severe anthracosis (Figure 1). No calcifications were observed. Lymphoid follicular hyperplasia and histiocyte infiltration have been reported as causes of increased FDG uptake in benign mediastinal LNs (3); thus, Glut-1 and CD-68 staining was performed in FP cases on PET/CT. However, immunohistochemical examination revealed equivocal Glut-1 staining in the lymph node cortex compared to that in positive control erythrocytes and CD-68-negative staining of the histiocytelike cells in the lymph node medulla. All patients with PET FP lymph nodes exhibited severe anthracosis (Tables 5,6).

\section{Comparison of characteristics between the false-positive and true-positive lymph nodes}

The clinical characteristics were compared between patients with FP and TP lymph nodes (Table 7). Six of eight FP cases had bilateral accumulations, whereas all six TP cases had unilateral accumulation $(\mathrm{P}=0.006)$. There was no significant difference in the size of the dissected PET FP and PET TP lymph nodes, but the short diameter on chest CT was significantly larger in PET TP lymph nodes $(\mathrm{P}=0.008)$. The median asbestos concentrations were 12,508/g dry lung (IQR: 9,666-94,474) in the FP group and 16,452/g dry lung (IQR: 7,448-65,249) in the TP group, with no significant difference between the groups $(\mathrm{P}=0.747)$. Similarly, there were no significant differences in smoking between the TP and $\mathrm{FP}$ groups $(\mathrm{P}=0.796)$.

\section{Discussion}

The present study explored the assessment of intrathoracic lymph node metastasis using FDG-PET/CT in patients with ARLC. Previous studies have proposed integrated PET/CT as a better modality for evaluating lymph nodes and distant metastasis (4-8). However, FDG-PET/CT has a high false-positive rate in the assessment of mediastinal lymph nodes in patients with lung cancer and those with some concomitant benign conditions such as infection, granulomatous lesions, and pneumoconiosis (9-12).

This study focused on occupational dust and asbestosrelated lung cancer. Asbestos is carcinogenic dust that causes pleural mesothelioma and lung cancer (13). Inhalation of asbestos results in a 50-fold increased risk of lung cancer among smokers (14). Silicosis and coalworkers pneumoconiosis are on a decreasing trend in 
Table 5 Characteristics of the false-positive lymph nodes

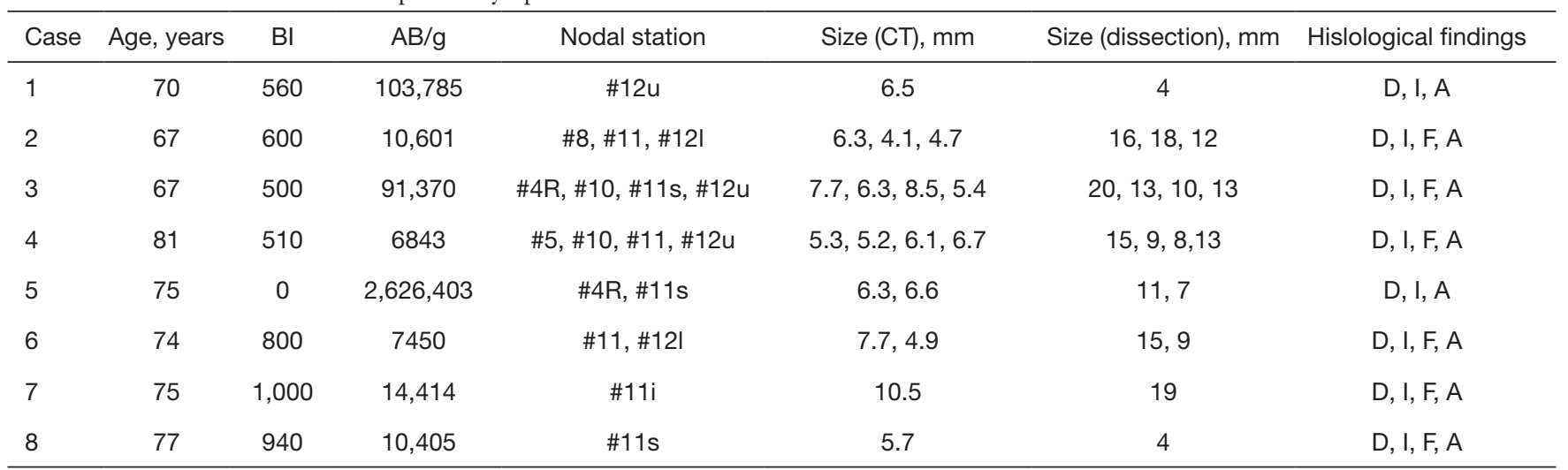

$\mathrm{BI}$, Brinkman index; size (CT), short axis diameter of lymph node on CT; size (dissection), long axis diameter of dissected lymph node; D, disruption of lymphoid follicles; I, infiltration of histiocyte-like cells; F, fibrous micronodules; A, severe anthracosis.

Table 6 Characteristics of the true positive lymph nodes

\begin{tabular}{lcccccc}
\hline Case & Age, years & $\mathrm{BI}$ & $\mathrm{AB} / \mathrm{g}$ & Nodal station & Size (CT), mm & Size (dissection), mm \\
\hline 1 & 84 & 750 & 80,384 & $\# 11 \mathrm{i}$ & 6.6 & 12 \\
2 & 82 & 550 & 123,486 & $\# 11 \mathrm{i}, \# 12 \mathrm{l}$ & $15.3,8.9$ & 9.3 \\
3 & 83 & 800 & 13,061 & $\# 11 \mathrm{~s}$ & 16 & 22 \\
4 & 67 & 690 & 5,577 & $\# 11$ & 14 & 12 \\
5 & 73 & 960 & 3,858 & $\# 11 \mathrm{~s}$ & $22,8,10,6$ \\
\hline
\end{tabular}

$\mathrm{BI}$, Brinkman index; size (CT), short axis diameter of lymph node on CT; size (dissection), long axis diameter of dissected lymph node.

Japan, but ARLC has a long latent period of about 20 years from asbestos exposure to the onset of disease (13). It is an occupational dust disease that will continue to increase in the future, although the use, manufacture, and import of asbestos were abolished in Japan in 2006. Nevertheless, the clinicopathological features of ARLC are not well known. Although previous studies have demonstrated the usefulness of PET/CT for the preoperative diagnosis of lung cancer, no studies have examined its sensitivity, specificity, or accuracy in ARLC. Thus, we conducted this study to explore the assessment of intrathoracic lymph node metastasis using FDG-PET/CT in patients with ARLC.

In the current study, the sensitivity, specificity, and accuracy of FDG-PET/CT for hilar and mediastinal lymph nodes in patients with ARLC were $71.4 \%, 87.8 \%$, and $86.3 \%$, respectively. In their meta-analysis, Pak et al. (5) reported that $\mathrm{PET} / \mathrm{CT}$ had a pooled sensitivity and specificity of $62 \%$ (widely ranging from $13 \%$ to $98 \%$ ) and $92 \%$ (ranging from $72 \%$ to $98 \%$ ), respectively. The accuracy of FDG-PET/CT in patients with ARLC was within the range reported in the literature. Regarding lung cancer with occupational dust diseases as the underlying disease, the sensitivity, specificity, and accuracy of PET/ $\mathrm{CT}$ in patients with lung cancer among coal workers were $84 \%, 65 \%$, and $74 \%$, respectively (15). The lymph node FP rate in patients with ARLC was lower than that in coal workers with lung cancer (12.2\% vs. 35\%). Regarding the cause of FP lymph nodes in patients with ARLC, there are no underlying diseases such as calcification of the lymph nodes, granulomatous diseases including tuberculosis, or pulmonary diseases such as interstitial pneumonia.

The histological findings of FP lymph nodes included disruption of lymphoid follicles in the cortex, infiltration of histiocyte-like cells, fibrous nodules, and severe anthracosis in the medulla. Shim et al. reported that FP lymph nodes showed calcification, follicular hyperplasia in the cortex, 

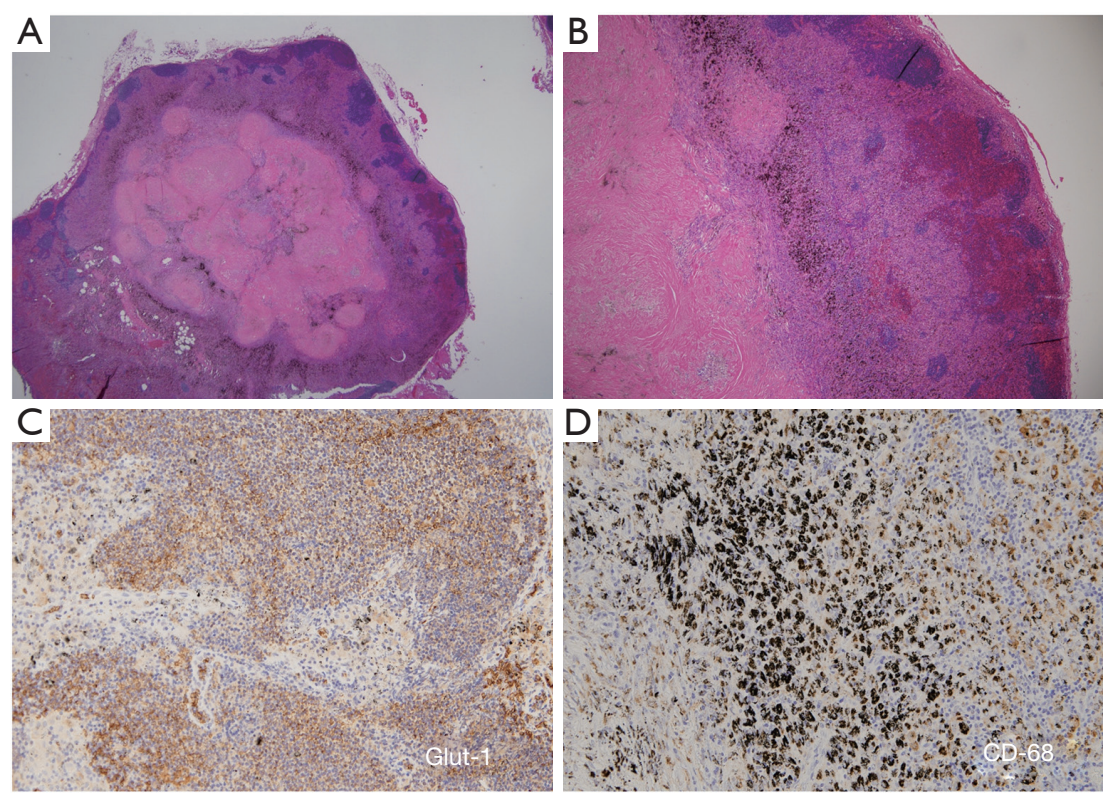

Figure 1 Histological findings of resected lymph nodes with PET false-positive results. (A) Histological section of a false-positive lymph node (H\&E staining, $\times 12.5$ ). (B) Histology showing fibrous nodule formation, anthracotic pigmentation in the medulla, and disruption of lymphoid follicles in the cortex (H\&E staining, $\times 40)$. (C) Immunostaining for GLUT-1 showing equivocal staining of the cortex area, except for the erythroblasts $(\times 200)$. (D) Histiocytic-like cells with anthracotic pigmentation showing CD-68-negative staining $(\times 200)$.

Table 7 Comparisons of patients with false-positive and true-positive lymph nodes

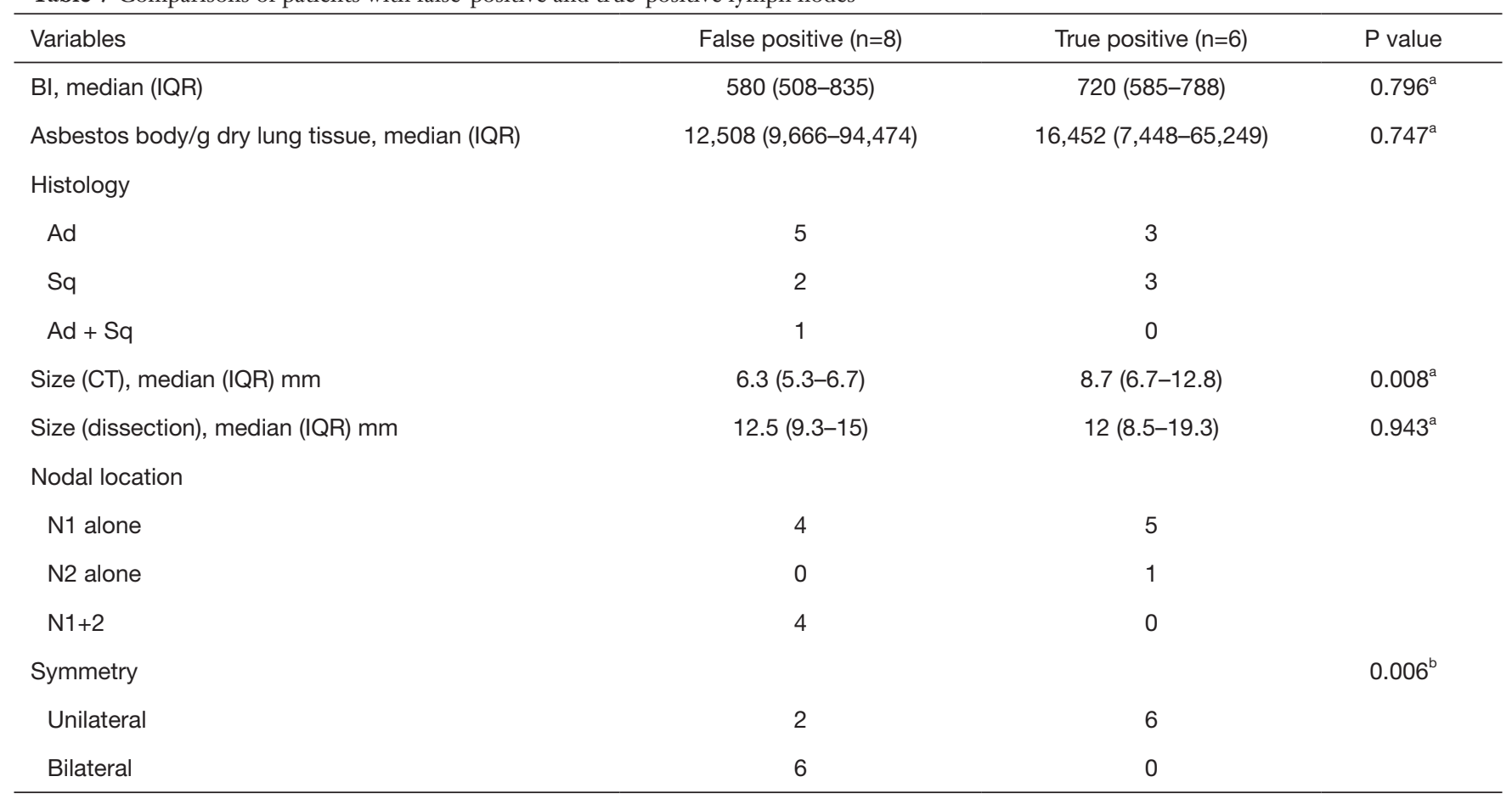

a, Mann-Whitney U test; ${ }^{b}$, Fisher's exact test. BI, Brinkman index; IQR, interquartile range; Ad, adenocarcinoma; Sq, squamous cell carcinoma; Ad + Sq, adenosquamous cell carcinoma; size (CT), short axis diameter of lymph node on CT; size (dissection), long axis diameter of dissected lymph node. 
macrophage infiltration with anthracotic pigmentation, and fibrotic nodule formation in the medulla (16). Calcification and lymphoid follicular hyperplasia were not observed in the present study. In addition, Glut-1 and CD 68 staining also revealed negative findings, although some studies have reported lymphoid follicular hyperplasia and histiocytic infiltration in FP nodes. Furthermore, Glut-1 overexpression is associated with FDG uptake $(3,17)$. The negative staining for Glut-1 may explain the disruption of lymphoid follicle in the cortex. Anthracosis and silicoanthracosis have been reported as other causes of falsepositive PET results in hilar and mediastinal lymph nodes (18-20). The occupational history of the eight patients with false-positive lymph nodes in these cases included four dockyards, one brickwork, one electrical work, one casting, and one pipework, all of whom had work environments where exposure to carbon dust inhalation was suspected in addition to asbestos inhalation. The main factor of FDG uptake in these cases may be related to severe anthracosis rather than lymphoid follicular hyperplasia or histiocytic infiltration.

FP lymph node findings affect treatment plans for lung cancer as mediastinal lymph node metastasis requires consideration of the operative indications, whether the primary treatment is surgery or not, while N1 lymph node metastasis requires consideration of the operative methods regarding the need for bronchoplasty or pulmonary angioplasty. In this study, six of eight FP lymph nodes had bilaterally symmetrical accumulations. The clinical features of FP lymph nodes in ARLC include bilaterally symmetric accumulations, similar to those in FP nodes in other diseases (21). Thus, the sensitivity and specificity can be improved and this method may be applied for the preoperative evaluation of lymph node metastasis, as in the case of ordinary lung cancer. This study has several limitations. Because ARLC is rare and there are few cases of FP lymph nodes, further case series are needed to investigate the histological features of FP lymph nodes in ARLC.

In our study, the sensitivity and specificity rates of FDG-PET/CT for ARLC for the assessment of hilar and mediastinal lymph node metastasis were $71.4 \%$ and $87.8 \%$, respectively. PET/CT scans of patients with ARLC had comparable sensitivity and specificity to those of PET/CT scans of patients with conventional lung cancer reported in the literature. Many FP cases also showed bilateral symmetric accumulation. This method can be used to evaluate lymph node involvement in lung cancer.

\section{Acknowledgments}

We would like to thank Editage (www.editage.com) for English language editing.

Funding: None.

\section{Footnote}

Reporting Checklist: The authors have completed the STROBE reporting checklist. Available at https://dx.doi. org/10.21037/jtd-21-974

Data Sharing Statement: Available at https://dx.doi. org/10.21037/jtd-21-974

Peer Review File: Available at https://dx.doi.org/10.21037/ jtd-21-974

Conflicts of Interest: All authors have completed the ICMJE uniform disclosure form (available at https://dx.doi. org/10.21037/jtd-21-974). The authors have no conflicts of interest to declare.

Ethical Statement: The authors are accountable for all aspects of the work in ensuring that questions related to the accuracy or integrity of any part of the work are appropriately investigated and resolved. This retrospective observational study was conducted in accordance with the Declaration of Helsinki (as revised in 2013), and approved by our institutional review board (approval no. 167; approval date: February 29, 2019). Informed consent was waived because of the anonymous nature of the data.

Open Access Statement: This is an Open Access article distributed in accordance with the Creative Commons Attribution-NonCommercial-NoDerivs 4.0 International License (CC BY-NC-ND 4.0), which permits the noncommercial replication and distribution of the article with the strict proviso that no changes or edits are made and the original work is properly cited (including links to both the formal publication through the relevant DOI and the license). See: https://creativecommons.org/licenses/by-nc-nd/4.0/.

\section{References}

1. Kishimoto T, Gemba K, Fujimoto N, et al. Clinical study of asbestos-related lung cancer in Japan with special reference to occupational history. Cancer Sci 
2010;101:1194-8.

2. Rusch VW, Asamura H, Watanabe H, et al. The iaslc lung cancer staging project: A proposal for a new international lymph node map in the forthcoming seventh edition of the tnm classification for lung cancer. J Thorac Oncol 2009;4:568-77.

3. Kwon SY, Min JJ, Song HC, et al. Impact of Lymphoid Follicles and Histiocytes on the False-Positive FDG Uptake of Lymph Nodes in Non-Small Cell Lung Cancer. Nucl Med Mol Imaging 2011;45:185-91.

4. De Leyn P, Dooms C, Kuzdzal J, et al. Revised ests guidelines for preoperative mediastinal lymph node staging for non-small-cell lung cancer. Eur J Cardiothorac Surg 2014;45:787-98.

5. Pak K, Park S, Cheon GJ, et al. Update on nodal staging in non-small cell lung cancer with integrated positron emission tomography/computed tomography: a metaanalysis. Ann Nucl Med 2015;29:409-19.

6. Chao F, Zhang H. PET/CT in the staging of the non-small-cell lung cancer. J Biomed Biotechnol 2012;2012:783739.

7. Gould MK, Kuschner WG, Rydzak CE, et al. Test performance of positron emission tomography and computed tomography for mediastinal staging in patients with non-small-cell lung cancer: a meta-analysis. Ann Intern Med 2003;139:879-92.

8. Bille A, Pelosi E, Skanjeti A, et al. Preoperative intrathoracic lymph node staging in patients with nonsmall-cell lung cancer: Accuracy of integrated positron emission tomography and computed tomography. Eur J Cardiothorac Surg 2009;36:440-5.

9. Li S, Zheng Q, Ma Y, et al. Implications of false negative and false positive diagnosis in lymph node staging of NSCLC by means of ${ }^{18} \mathrm{~F}-\mathrm{FDG}$ PET/CT. PLoS One 2013;8:e78552.

10. Lee J, Kim YK, Seo YY, et al. Clinical Characteristics of False-Positive Lymph Node on Chest CT or PETCT Confirmed by Endobronchial Ultrasound-Guided Transbronchial Needle Aspiration in Lung Cancer. Tuberc Respir Dis (Seoul) 2018;81:339-46.

11. Konishi J, Yamazaki K, Tsukamoto E, et al. Mediastinal lymph node staging by FDG-pet in patients with nonsmall cell lung cancer: Analysis of false-positive FDG-pet findings. Respiration 2003;70:500-6.

12. Roberts PF, Follette DM, von Haag D, et al. Factors associated with false-positive staging of lung cancer by positron emission tomography. Ann Thorac Surg
2000;70:1154-9; discussion 1159-60.

13. Prazakova S, Thomas PS, Sandrini A, et al. Asbestos and the lung in the 21st century: an update. Clin Respir J 2014;8:1-10.

14. Klebe S, Leigh J, Henderson DW, et al. Asbestos, Smoking and Lung Cancer: An Update. Int J Environ Res Public Health 2019;17:258.

15. Saydam O, Gokce M, Kilicgun A, et al. Accuracy of positron emission tomography in mediastinal node assessment in coal workers with lung cancer. Med Oncol 2012;29:589-94.

16. Shim SS, Lee KS, Kim BT, et al. Non-small cell lung cancer: prospective comparison of integrated FDG PET/ CT and CT alone for preoperative staging. Radiology 2005;236:1011-9.

17. Chung JH, Cho KJ, Lee SS, et al. Overexpression of Glut1 in lymphoid follicles correlates with false-positive (18) F-FDG PET results in lung cancer staging. J Nucl Med 2004;45:999-1003.

18. Toba H, Kondo K, Otsuka H, et al. Diagnosis of the presence of lymph node metastasis and decision of operative indication using fluorodeoxyglucose-positron emission tomography and computed tomography in patients with primary lung cancer. J Med Invest 2010;57:305-13.

19. Lin WY, Hsu WH, Lin KH, et al. Role of preoperative PET-CT in assessing mediastinal and hilar lymph node status in early stage lung cancer. J Chin Med Assoc 2012;75:203-8.

20. Koksal D, Demirag F, Bayiz H, et al. The correlation of SUVmax with pathological characteristics of primary tumor and the value of Tumor/ Lymph node SUVmax ratio for predicting metastasis to lymph nodes in resected NSCLC patients. J Cardiothorac Surg 2013;8:63.

21. Lee JW, Kim BS, Lee DS, et al. 18F-FDG PET/CT in mediastinal lymph node staging of non-small-cell lung cancer in a tuberculosis-endemic country: consideration of lymph node calcification and distribution pattern to improve specificity. Eur J Nucl Med Mol Imaging 2009;36:1794-802.

Cite this article as: Iga N, Sonobe $\mathrm{H}$, Mizuno D, Nishi $\mathrm{H}$. Assessment of intrathoracic lymph nodes by FDG PET/CT in patients with asbestos-related lung cancer. J Thorac Dis 2021;13(10):5925-5932. doi: 10.21037/jtd-21-974 